\title{
LA QUÍMICA COMO UN PROGRAMA DE INVESTIGACIÓN CIENTÍFICA Un ejemplo a través de la teoría de Lewis
}

\author{
THE CHEMISTRY A SCIENTIFIC RESEARCH PROGRAM
}

An example with the Lewis Theory

Leonardo Fabio Martínez*

Manuel Fredy Molina Caballero**

\begin{abstract}
Resumen
En el presente trabajo se discute si la química es un programa de investigación científica. Tomando como perspectiva el análisis histórico, se muestra la teoría de Lewis y algunos de sus contemporáneos como una construcción comunitaria que se constituye como un referente de la química como ciencia.

Palabras clave: Programa de investigación científica, historia y epistemología de la química, teoría atómica, teoría del enlace químico.
\end{abstract}

\begin{abstract}
The purpose of this paper is to discus if chemistry is a scientific and research program from the historical and epistemogical perspective. Lewis chemical bond theory is an example of the work done by a group under the concept of chemistry as a science.
\end{abstract}

Key words: Research program scientific, history and epistemology of the chemistry, atomic theory, chemical bond theory.

\footnotetext{
*Profesor Departamento de Química. Universidad Pedagógica Nacional. lemartinez@uni.pedagogica.edu.co.

** Grupo de Docencia de la Química. Universidad Nacional de Colombia. mfmolinac@unal.edu.co
} 


\section{INTRODUCCIÓN}

El presente ensayo se enmarca en el campo de la discusión epistemológica, tomando como perspectiva el análisis histórico, para el caso de la construcción y desarrollo de la teoría de Lewis (1916).

Se discute a manera de ejemplo la teoría de Lewis, para mostrar un ejercicio intelectual de la comunidad de especialistas en química como un programa de investigación en los términos de Lakatos, I. (1983).

En este orden de ideas, es importante señalar que en la última década del siglo $X X$, la investigación didáctica ha demostrado la importancia que tienen la historia y la epistemología para la enseñanza de las ciencias de la naturaleza y en particular para la enseñanza de la química. El trabajo de Lombardi (1997) contribuye a una valoración significativa de los estudios históricos en oposición a los estudios historiográficos. La diferencia fundamental entre la historia y la historiografía está en un reconocimiento a la dinámica de la comunidad de especialistas de un determinado momento histórico, que llevó a la producción de teorías o modelos científicos que satisfacen los problemas planteados por la comunidad; esta nueva concepción de la historia supera la presentación lineal de algunos trabajos que presentan un conjunto de hechos y autores sin ninguna articulación.

La historia y epistemología de las ciencias posibilita comprender la ciencia como una producción cultural (Castro, 2003).
Producción cultural sujeta a las diferentes tensiones y discusiones que permiten el establecimiento de un programa de investigación científica. La anterior perspectiva abre un campo de investigación cuya preocupación es reconstruir las ciencias de la naturaleza desde los autores que han aportado a su desarrollo. Los trabajos en este campo proporcionan valiosos elementos para la didáctica de las ciencias, especialmente se han destacado los estudios de los libros de texto, por ejemplo, Libres; Comesaña y Toro, (2001) han demostrado una escasa presencia de la historia en los libros de texto, circunstancia que puede propiciar apreciaciones equivocadas frente a la actividad científica.

Ahora bien, este ensayo comprende dos partes. En la primera parte se realiza una reflexión acerca de la ciencia como una construcción histórica y comunitaria, coherente con la propuesta de Lakatos, y en la segunda parte se analiza la teoría del enlace químico, que es sostenida por Lewis como un claro ejemplo de un programa de investigación en química.

\section{LOS PROGRAMAS DE INVESTIGACIÓN CIENTÍFICA}

Para este trabajo se toma como perspectiva de análisis los aportes teóricos del filósofo de las ciencias Lakatos (1983), quien realiza una crítica a la ingenuidad del criterio de demarcación de Popper para distinguir un conocimiento científico de uno pseudocientífico. Lakatos señala en este sentido que no basta con asegurar que si un saber es falsable es científico. Contrario a esto, Lakatos sustenta que dentro de 
una propuesta científica o un paradigma existe una fuerte resistencia al cambio, por esto la comunidad se esfuerza en no abandonar los acuerdos establecidos en el paradigma.

Desde la postura de Lakatos, la ciencia estaría constituida por programas de investigación científica (PI) que poseen un núcleo firme, en donde se encuentran los principios básicos y un cinturón de hipótesis que protegen dicho núcleo. Puede suceder que en un programa las hipótesis protectoras se vean seriamente cuestionadas, pero que su núcleo se mantenga firme con la creación de nuevas hipótesis que resuelvan los cuestionamientos planteados; así mismo, dichos programas de investigación gozan de un apoyo empírico, que soporta tanto las hipótesis protectoras como el núcleo firme del programa de investigación científica.

El trabajo de Lakatos ha sido valorado por varios investigadores, entre ellos Estany (1990), quien sustenta que Lakatos construye una propuesta de análisis histórico de la ciencia que supera el falsacionismo que no logra dar cuenta del cambio científico, ya que el criterio de falsación no puede ser usado para explicar las rupturas paradigmáticas de la ciencia, que son explicadas adecuadamente a partir de la historia de las ciencias, que proporciona evidencia para validar científicamente un cuerpo teórico.

Para comprender la dinámica del cambio científico, Lakatos construye dos elementos esenciales: el primero, que fue previamente presentado, corresponde a los $\mathrm{PI}$ reconocidos y valorados por la tradición y renovación de una comunidad en cuanto al núcleo firme del programa y su respectivo cinturón protector. El segundo elemento es de carácter metodológico y consiste en la heurística negativa y la heurística positiva.

La heurística negativa de un PI impide que el núcleo firme sea abandonado. Para ello, recurre a fortalecer el cinturón protector conformado por hipótesis, prepusupuestos y construcciones empíricas que garantizan la consistencia del núcleo, esto no va acompañado necesariamente de la reformulación de los aspectos esenciales del cinturón protector, lo cual da cuenta de su desventaja en relación con la heurística positiva.

Por su parte la heurística positiva garantiza que los investigadores de un PI busquen la manera de solucionar las anomalías que pueda presentar el cinturón protector, que en situaciones drásticas implica el cambio del cinturón mediante la construcción de modelos que renueven el programa y garanticen su continuidad. Un claro ejemplo de esta situación es presentado por Lakatos (1983) cuando se refiere al PI de Newton:

En principio Newton elaboró su programa para un sistema planetario con un punto fijo que representaba al sol y un único punto que representaba a un planeta. A partir de este modelo derivó su ley de inverso del cuadrado para la elipse de Kepler, pero este modelo contradecía a la tercera ley de la dinámica de Newton y por ello tuvo que ser sustituido por otro 
en que tanto el sol como el planeta giraban alrededor de su centro de gravedad común.

La heurística es una herramienta metodológica importante para comprender la dinámica histórica del PI incluso cuando este es abandonado por sus defensores, permitiendo comprender cuán grande era su capacidad para explicar sus propias refutaciones en el curso de crecimiento, cuántos hechos produjeron, qué del programa ha sido retomado y que se ha olvidado.

Teniendo en cuenta estos aspectos, a continuación se discute el trabajo de Lewis, Gilbert Newton (ver figura 1) en el programa de investigación científica de la química.

\section{LA QUÍMICA DESDE LA PERSPECTIVA DE PROGRAMAS DE INVESTIGACIÓN}

Siguiendo la perspectiva de Lakatos, la química es una ciencia constituida por programas de investigación científica que han sido redefinidos en el transcurso de la historia. Un ejemplo claro de esta perspectiva, como ya fue mencionado, puede ser la teoría de enlace químico de Lewis (1916), que aún es usada para explicar la formación de algunas moléculas, aunque haya sido superada y rebatida por físicos y químicos, tales como Pauling, Heitler y London.

Lewis, teniendo en cuenta los trabajos de periodicidad química y valencia, comenzó a formular su teoría atómica y con ella su teoría del enlace químico.

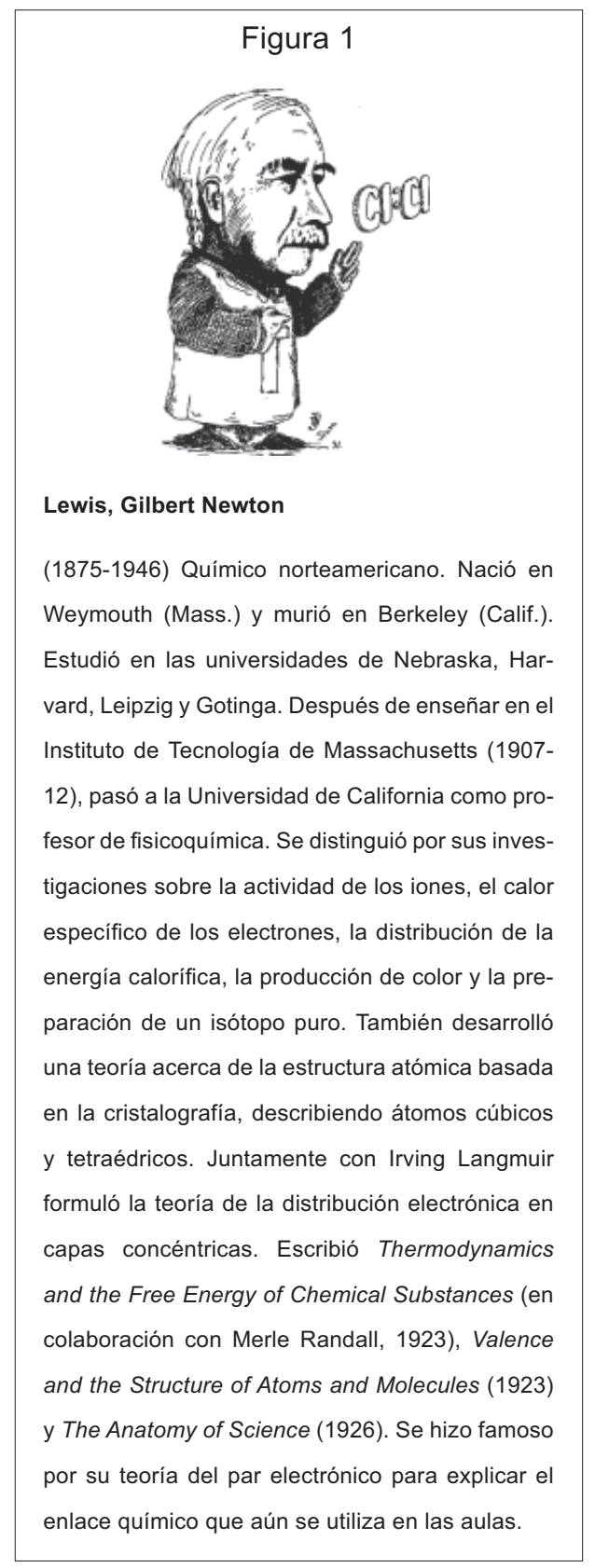

Tomado de: http://www.woodrow.org/teachers/ci/1992/ LEWIS.GIF

A principios del siglo $\mathrm{XX}$, en la comunidad de químicos llamaba la atención el 
comportamiento de los gases nobles. Al parecer estas sustancias eran tan estables que se llegó a pensar que eran inertes. Sin embargo, esta idea fue debatida y posteriormente cambiada. Por otra parte, es clara la relación entre el número atómico y la manera como se distribuían los electrones en el átomo; al mismo tiempo que se sabía que dicho número atómico asociado al peso atómico guardaba estrecha relación con las propiedades químicas de familias de elementos. Esto se refería en buena medida a la periodicidad química.

Lewis, sintetizando los aportes de los químicos, empezó a construir un modelo de átomo y una representación del enlace químico, de acuerdo a su evidencia empírica y sus construcciones explicativas; él se apartó significativamente de la ideas de los físicos, que buscaban dar cuenta de la estructura atómica en consonancia con principios físico-matemáticos clásicos, tales como la ley de Coulomb.

\section{¿Por qué surgió la teoría de Lewis?}

En el siglo XIX se concebía a la materia constituida por átomos que deberían estar unidos de distintas formas. Con los trabajos de Alessandro Volta (17451827), se atribuyó la unión a la fuerza eléctrica, lo que se demostró de manera inversa cuando William Nicholson (17531815) y Anthony Carslile (1768-1840) mostraron que la corriente eléctrica separaba los compuestos en sus átomos. Como ejemplo, el agua se separaba en hidrógeno y oxígeno. Humphry Davy
(1778-1829) siguió esta línea de trabajo, y en 1811 Berzelius dio un gran impulso a este pensamiento, postulando una teoría de atracción eléctrica, imaginando dos cargas, positiva y negativa, representando el enlace como la neutralización de las cargas. Svante Arrhenius (18591927) argumento, desde los experimentos electrolíticos con soluciones acuosas, que la atracción entre átomos se debía a fuerzas eléctricas, idea apoyada por Helmholtz (1821-1894).

La amplia aplicación de estas teorías empezó a fallar cuando se quiso aplicar a los nuevos compuestos orgánicos, que comenzaron a obtenerse a finales del siglo XIX. Y resultó inadecuado aplicar el modelo de cargas eléctricas a estos compuestos.

El enlace en compuestos orgánicos no recibió exitosa interpretación hasta cuando en 1916 Lewis anunció la teoría de la compartición de pares de electrones. Lewis mostró que la formación de compuestos con atracciones eléctricas es un caso especial de una teoría más general (Stranges, 1982).

\section{¿Cuál es el sustento de la teoría de Lewis?}

En 1897, Thomson (1856-1940) demostró, con un experimento de conductividad eléctrica en gases, la existencia de los electrones, y que son responsables de la atracción eléctrica. Thomson argumentaba que las atracciones entre átomos se debían a la donación de un electrón al segundo átomo y este se cargaba negativamente, mientras el otro lo hacía positivamente. 
El alemán Richard Abbeg (1869-1910) en 1904 desarrolló una teoría electrostática del enlace atómico. Tomó la regla del octeto como una indicación de número máximo de electrones envueltos en una unión atómica. Abegg observó que el mismo átomo en compuestos diferentes suma ocho entre cargas positivas y negativas. Por ejemplo en el $\mathrm{NaCl}$, el cloro tiene - 1 (para esta época el símbolo se presentaba adelante, actualmente es lo contrario) y en $\mathrm{HClO} 4$ tiene +7 ; $\mathrm{N}$ en $\mathrm{NH} 3$ $-3 y+5$ en HNO3. La regla de Abegg fue la primera publicación relacionada con el número de electrones en un enlace (electrones de valencia) (Pauling, 1984).

Teniendo en cuenta los antecedentes mencionados, Lewis, en su artículo, publicado en 1916, "The Atom and the Molecule", comienza discutiendo la clasificación tradicional de moléculas como orgánicas e inorgánicas, considerando que es más adecuado organizar las moléculas en polares y no polares. Aunque muchas moléculas orgánicas son consideradas como polares y las inorgánicas como no polares, se pueden evidenciar comportamientos químicos de algunas moléculas orgánicas que dan cuenta de su polaridad.

El criterio de polaridad está expresado en consonancia con ciertas propiedades químicas, tales como: reactividad, ionización, tautomerismo, isomerismo, entre otras. Lewis comienza a construir su explicación de la polaridad a partir de la manera como se distribuyen los electrones en el átomo, lo cual determinaría la representación de la carga.

\section{¿Cuál fue la contribución de Lewis?}

Asumió que cuando dos átomos se combinan, los dos átomos comparten un par de electrones entre ellos. Esto eliminó la existencia de cargas opuestas en los átomos en compuestos cuyas propiedades físicas y químicas no las indicaban.

El cambio de concepción, como se ilustra en el $\mathrm{HCl}$ la unión química:

\section{$\mathrm{H}^{+}-\mathrm{Cl}$ Teoría cargas positiva-negativa H:Cl Teoría de Lewis}

Para los compuestos polares Lewis sugirió que por cada enlace formado entre dos átomos en un compuesto polar, uno de los átomos atrae mucho más fuertemente los electrones que el otro, $y$ el caso extremo es la pérdida del par de electrones.

La razón por la que Lewis enuncia la compartición del par de electrones está sustentada en la información que tenían los químicos de muchos compuestos que contenían un número par en la suma de los electrones totales de valencia;

$$
\mathrm{NH}_{3}=8 \mathrm{CH}_{4}=8 \quad \mathrm{CO}_{2}=16
$$

Lewis mostró que fácilmente podría armar estructuras combinando átomos que compartieran pares de electrones. Esto satisface la representación de numerosos compuestos, como la del cloruro de amonio, oxiácidos y de hidruros metálicos.

Lewis propuso una nueva forma de simbolizar las estructuras, que hoy en día se conoce como formulas de Lewis. Estas representaciones se adoptaron rápida- 
mente casi universalmente. El más grande exponente de la teoría de Lewis fue Irving Lagmuir (1881-1967), que escribió varios libros donde utilizaba la teoría.

Esto llevó a crear confusiones en cuanto al autor de la teoría, que algunas veces llamaban de Lewis-Lagmuir. Para aclarar dudas, en 1923 Lewis publicó su monografía titulada Valence and the Structure of Atoms and Molecules. (Stranges, 1984).

Teniendo en cuenta los anteriores planteamientos y especialmente los aportes de Abegg en cuanto a la valencia para explicar la formación de moléculas, Lewis elabora su concepción de átomo cúbico.

Sustentado en la estabilidad de las moléculas Lewis concibió al átomo como un cubo. Para él, dicha estabilidad se debía a la distribución geométrica de los electrones en el átomo; a Lewis no le preocupaba, en cierta medida, la representación del núcleo atómico ni su incidencia en la molécula; para él, existía una región central en el átomo, denominada Kernel, en donde la estabilidad era evidente; dada la compensación de las atracciones y repulsiones entre cargas positivas y negativas, incluso los electrones que estaban cerca al Kernel (electrones de core) no eran importantes en el momento de formación de una molécula, sólo importaban los electrones externos; de esta manera Lewis graficó varios átomos, como el oxígeno, el litio, el carbón, el flúor, entre otros, tal como se muestra en la figura 2.

Figura 2

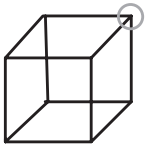

$\mathrm{Li}$

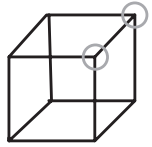

$\mathrm{Be}$

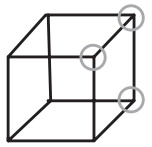

B

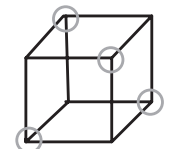

$\mathrm{C}$

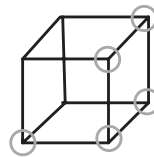

$\mathrm{N}$

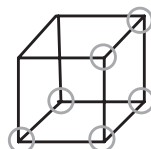

$\mathrm{O}$

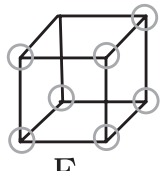

$\mathrm{F}$

Representación hecha por Lewis, de diferentes átomos. Tomado de: The Atom and the Molecule. Journal of the American Chemical Society Volume 38, 1916, pages 762-786. En www. Dbhs.wvusd.k12.ca.us/Chem-History

Así, Lewis representó los electrones del átomo de diferentes elementos en los vértices del cubo, en el caso de los gases nobles, los vértices del cubo estaban completamente ocupados, lo cual explicaba geométricamente la estabilidad de dichos elementos; aquellos elementos que no tenían ocupados en su totalidad los vértices del cubo tendían a alcanzar la organización electrónica de los gases nobles, com- partiendo electrones con otros átomos; no obstante, los arreglos de las moléculas se debían a la compartición de un par de electrones que incluso para algunas moléculas constituía su estabilidad.

El núcleo firme de su teoría (el par electrónico) lo establece formulando unos principios fundamentales. Los postulados de la teoría se pueden describir de la siguiente forma: 
1. En cada átomo existe una parte que no es alterada cuando se realiza un cambio químico, a esta capa se la denomina Kernel, dicha región presenta una carga neta positiva.

2. Además del Kernel, el átomo contiene una capa externa en la que una molécula neutraliza la carga neta positiva. Esta capa está compuesta por electrones $y$, en el caso de que transcurra una reacción química, el número de electrones puede variar de cero a ocho electrones.

3. En una combinación el átomo tiende a poseer en la capa externa un número par de electrones, que en varios casos pueden ser ocho. Estos se arreglan en los vértices del cubo.

4. Las dos capas del átomo son impenetrables entre ellas.

5. Los electrones que se encuentran en la capa externa (electrones de valencia) pueden moverse con facilidad. Esto depende de la naturaleza del átomo y del cambio químico que acontezca.

6. Las fuerzas eléctricas que se presentan entre las capas del átomo no obedecen a la ley que indica que las fuerzas de atracción o de repulsión entre dos cargas es proporcional al producto de las cargas e inversamente proporcional al cuadrado de su distancia.

Bajo estos principios, Lewis estructuró el cinturón protector de su teoría. No obstante, hay que decir que no pasó mucho tiempo para empezar a ser cuestionado, claro ejemplo de esto se evidencia en la explicación de la geometría para el áto- mo de carbono que, según los estudios en estereoquímica de Le Bel y Van't Hoff, presentaba una organización tetraédrica; para dar cuenta de esto, Lewis intentó modificar parte del cinturón protector, que estaba conformado por sus trabajos frente a la polaridad de las moléculas, asunto del que posteriormente no logra dar cuenta y comienza a derrumbar sus principios básicos; lo anterior se puede justificar apoyados En Cruz, D.; Chamizo, J., y Garrita, A. (1987), que citan a Lewis con respecto al problema del átomo de carbono:

Cuando consideramos únicamente fenómenos químicos, así como su mejor interpretación en términos de la estructura atómica, debemos asumir un arreglo diferente del grupo de los ocho electrones, al menos en el caso de sustancias no polares, cuyas moléculas generalmente están compuestas de átomos de volumen pequeño.

El programa de investigación es retomado en cierta parte por Langmuir (1919) que al principio de su artículo "The structure of atoms and the octet theory of valence", considera que da a conocer una nueva teoría del enlace químico, guiado por los datos químicos, que a su vez, es una extensión de la teoría de Lewis del átomo cúbico y la propuesta atómica de Rutherfor. Aunque hay que aclarar que la contribución de Langmuir es un intento de sintetizar los aportes de los físicos y químicos en una teoría, en muchos apartes de su propuesta se reflejan principios del núcleo firme de la teoría de Lewis. 
Lagmuir considera que la organización molecular más estable y simétrica corresponde a la de los gases "inertes", afirmación expuesta previamente por Lewis. Dicha estabilidad se puede explicar por la existencia de fuertes campos de atracción interna y débiles campos de atracción externa; además, Lagmuir consigue relacionar esto con el peso atómi$\mathrm{co}$, ya que cuando es menor al parecer el campo externo es más débil; así mismo, asume que la organización electrónica más estable es la que corresponde al par de electrones que posee el átomo de Helio. No obstante, el aporte más significativo de Lagmuir tiene que ver con su teoría del octeto, extensión de la propuesta de los ocho electrones de Lewis; su teoría del octeto brinda una respuesta a la organización electrónica de moléculas que poseen más de un par de electrones.

Desde la perspectiva de la teoría del octeto, el arreglo más estable es el que puede ser representado por ocho electrones; con base en esto propuso una regla aritmética $(e=8 n-2 p$, en donde $p$ representaría el número de enlaces covalentes) que podía predecir la existencia de compuestos químicos, y en 1919 también utilizó el termino de enlace covalente para reemplazar al antes utilizado "enlace de pares compartidos".

Los aportes teóricos de Lewis y Lagmuir para la química, como programa de investigación científica, son usados en nuestros días y sirven para explicar buena parte de los enlaces químicos de las moléculas -especialmente las orgánicas-; sin embargo, no da cuenta de otras preguntas, tales como: la geometría molecular, el paramagnetismo, los valores de energía de enlace y de ángulos de enlace que presentan la moléculas, cuestionamientos que serían abordados con mayor poder holístico desde las teorías de repulsión de pares electrónicos, la teoría del enlace de valencia y la teoría del orbital molecular.

\section{¿Por qué aún se utiliza la teoría de Lewis?}

En 1925, Pauli (1900-1958) enuncio el principio de exclusión, de acuerdo al cual dos electrones no podrían tener iguales los cuatro números cuánticos, sino que se diferenciaban en su número de espín, que les daba una orientación opuesta a cada uno. En otras palabras, los dos electrones se complementaban, formaban un par. Pauli aplicó la hipótesis del par electrónico al enlace químico y fue correspondiente con lo propuesto por Lewis.

Linus Pauling justificó teóricamente la regla del octeto con moléculas como $F_{2}$, $\mathrm{Cl}_{2}$ y $\mathrm{CH}_{4}$. Además, propuso la teoría del enlace de valencia que considera el par electrónico como un elemento esencial para explicar la estructura molecular.

La mecánica cuántica ha asumido la teoría del par electrónico de Lewis dando explicaciones matemáticas, la más conocida es la de la molécula de $\mathrm{H} 2$ y por ello hoy en día la teoría de Lewis es aceptada y trabajada en las aulas, aunque más su modelo de presentación del enlace.

La utilización del par electrónico desde el punto de vista de interpretación 
electrostática sirve para construir un modelo de enlace como una atracción de electrones por dos núcleos, lo que confiere estabilidad al sistema. El mayor éxito de la teoría fue incorporarse a la Teoría de Repulsión de Pares Electrónicos en la Capa de Valencia, donde ayuda a predecir bien las geometrías electrónicas y moleculares de las moléculas partiendo de la simple notación de Lewis para los enlaces químicos.

Desde el punto de vista didáctico, la forma de escritura del enlace químico de Lewis es un buen modelo, que permite visualizar macroscópicamente lo que ocurre microscópicamente, permitiendo por medio de símbolos relacionar el mundo atómico con el mundo tangible, logrando así que un alumno comprenda un proceso que jamás verá en la realidad. El modelo de escritura de puntos de Lewis se adapta muy bien cuando se desea ilustrar lo que pasa con los electrones al formarse un enlace químico. Este modelo de escritura no dice nada acerca de qué es un enlace químico o cómo se forma, y por ello permanece ante cualquier cuestionamiento; además, se cumple correctamente con los primeros 20 elementos $(\mathrm{H}$ al $\mathrm{Ca}$ ), que sin duda representa la mayor cantidad de compuestos químicos (solo habría que pensar en los compuestos de $\mathrm{C}, \mathrm{H}, \mathrm{O}$ y $\mathrm{N}$ ).

\section{Comentarios finales}

A lo largo de la discusión del texto, se ha sustentando que la química puede ser considerada como un programa de investigación científica, construido por un ejercicio comunitario; así se ilustra en el programa de investigación la teoría atómica de Lewis como un esfuerzo intelectual que logró constituir un núcleo firme de trabajo y un cinturón protector. Este programa hace parte de la construcción de la química para principios del siglo XX.

Además, se debe señalar que Lewis desarrolla la teoría del enlace químico que explica la manera como los átomos se unen para formar moléculas gracias a sus electrones de valencia; dicha teoría fue extendida por científicos de la época, como Langmuir, que construye una expresión matemática para calcular los enlaces covalentes que presenta una molécula. Así la propuesta de Lewis se prolonga a través de los años e incluso es usada en la actualidad por varios químicos para explicar la formación de las moléculas. Esto constituye un ejercicio histórico y comunitario que es claro ejemplo de un desarrollo histórico para la química como programa de investigación científica.

No obstante, hay que señalar que la teoría de Lewis, como parte de un programa de investigación científica, ingresa a un periodo de regresión cuando no logra explicar ciertos fenómenos del enlace químico, como la geometría molecular y el paramagnetismo de algunas moléculas.

Finalmente se sostiene que a pesar que la teoría de Lewis, ha sido seriamente cuestionada por las teorías del enlace hechas desde la cuántica se constituye en un ejercicio intelectual importante, que incluso hoy sobrevive y que sirve para re- 
presentar muchas moléculas; más aún, los planteamientos de Lewis son retomados por las investigaciones de Pauli con el principio de exclusión y por Pauling en el desarrollo de su teoría de enlace de valencia, lo que confirma que las teorías

\section{Biblografía}

Castro, J. (2003). La filosofía y la historia de las ciencias en los libros de texto. Un análisis de la teoría evolutiva. En Tecné, Episteme y Didaxis, No. extra, pp. 213-215.

Cruz, D.; Chamizo, J., y Garritz, A. (1987). Estructura Atómica: un enfoque químico. USA: Addison Wesley Iberoamericana.

Dausel, R. (1975). Teoría cuántica del enlace químico. España: Bellaterra.

Erazo, M. (1999). Caracterización de la influencia empiropositivista que guía el pensamiento de los profesores de ciencias. Bogotá: Universidad Pedagógica Nacional.

Estany, A. (1990). Modelos de Cambio Científico. España: Crítica.

Gallego, R.; Pérez, R., y Torres de Gallego, L. (1996). La química como ciencia: Una perspectiva constructivista. En Estudios en pedagogía y didáctica. 1(2), pp. 6-16.

Keith J., Laidler. (1993). The World of Physical Chemistry. USA: Oxford New York.

Langmuir, I. (1919). The Structure of atoms and the octet theory of valence. En: Prodeedings of the National Academy of Science Vol. V, 252.www. Dbhs.wvusd.k12.ca.us/ Chem-History

Lakatos, I. (1983). La Metodología de los programas de investigación científica. Madrid: Alianza. construidas en un programa de investigación científica no son ingenuamente olvidadas, sino que se extienden en el tiempo con desarrollos teóricos diversos, que la comunidad científica construye.

Lewis, G. (1916). The Atom and the Molecule. En Journal of the American Chemical Society. Volume 38, 1916, pp. 762-786. www. dbhs.wvusd.k12.ca.us/

Libres, M.; Comesaña, M., y Y Toro, J. (2001). La historia de la química en los libros de texto: una escasez escandalosa. En Enseñanza de las ciencias. No. Extra, pp. 243 -244.

Lombardi, O. (1997). La pertinencia de la historia en la enseñanza de ciencias. Argumentos y contraargumentos. En Enseñanza de las ciencias 15 (3), pp. 343 -349.

Pauling, L. (1984). G. N. Lewis and the Chemical Bond. En Journal of Chemical Education, 61(3), pp. 201-203.

Stranges, A. (1982). Electrons and valence. Texas: University Press.

Stranges, A. (1984). Reflections on the Electrón Theory of the Chemical Bond: 19001925. En Journal of Chemical Education, 61(3), pp. 185-190.

Romero, O., y Yánez, M. (2000). Enlace químico y estructura molecular. España: Bosch Editor.

Villaveces, J.; Poveda, F., y Cubillos, G. (1989). Hacia una historia epistemológica de la química. Bogotá: Academia Colombiana de Ciencias Exactas.

ArTículo RECIBIDO: 19-11-2004

Y APROBADO: 17-06-2005 